\title{
Asymmetric localization of natural antisense RNA of neuropeptide sensorin in Aplysia sensory neurons during aging and activity
}

\author{
Beena M. Kadakkuzha ${ }^{\dagger}$, Xin-An Liu ${ }^{\dagger}$, Maria Narvaez, Alexandra Kaye, Komolitdin Akhmedov and \\ Sathyanarayanan V. Puthanveettil *
}

Department of Neuroscience, The Scripps Research Institute, Jupiter, FL, USA

\section{Edited by:}

Yingqun Huang, Yale University

School of Medicine, USA

\section{Reviewed by:}

Tohru Yoshihisa, University of

Hyogo, Japan

Alessio Paone, Sapienza University

of Rome, Italy

\section{*Correspondence:}

Sathyanarayanan V. Puthanveettil, Department of Neuroscience, The Scripps Research Institute, Scripps Florida, 130 Scripps way, Jupiter,

FL 33458, USA

e-mail: sputhanv@scripps.edu

${ }^{t}$ These authors have contributed equally to this work.

\begin{abstract}
Despite the advances in our understanding of transcriptome, regulation and function of its non-coding components continue to be poorly understood. Here we searched for natural antisense transcript for sensorin (NAT-SRN), a neuropeptide expressed in the presynaptic sensory neurons of gill-withdrawal reflex of the marine snail Aplysia californica. Sensorin (SRN) has a key role in learning and long-term memory storage in Aplysia. We have now identified NAT-SRN in the central nervous system (CNS) and have confirmed its expression by northern blotting and fluorescent RNA in situ hybridization. Quantitative analysis of NAT-SRN in micro-dissected cell bodies and processes of sensory neurons suggest that NAT-SRN is present in the distal neuronal processes along with sense transcripts. Importantly, aging is associated with reduction in levels of NAT-SRN in sensory neuron processes. Furthermore, we find that forskolin, an activator of CREB signaling, differentially alters the distribution of SRN and NAT-SRN. These studies reveal novel insights into physiological regulation of natural antisense RNAs.
\end{abstract}

Keywords: memory, nocoding RNA, antisense RNA, aging, Aplysia, neural circuitry

\section{INTRODUCTION}

Recent high-throughput transcriptome studies have revealed widespread and extensive overlaps between genes and transcripts encoded on both strands of the genomic sequence. This overlapping gene organization, which produces sense-antisense transcript pairs, is capable of affecting regulatory cascades through established mechanisms. Natural antisense transcripts (NATs) are transcribed from the opposite strand to a protein coding or sense strand in the chromatin. Recent studies have provided ample evidence that more than $70 \%$ of the mammalian genome have antisense transcription potential.

Antisense transcription has been recognized for roles in gene regulation involving degradation of the corresponding sense transcripts (RNA interference), as well as gene silencing at the chromatin level (Faghihi and Wahlestedt, 2009; Pelechano and Steinmetz, 2013). Gene expression profiling studies show frequent concordant regulation of sense-antisense transcript pairs though there is clear evidence of discordant regulation, leading to significant physiological outcomes such as neurodegenerative diseases (Kadakkuzha et al., 2013). It has been shown that experimental modulation of an antisense transcript RNA can change the expression of sense transcript, supporting the role of antisense transcription to control of transcriptional outputs in higher animals (Katayama et al., 2005; Modarresi et al., 2011).

Despite efforts to unravel the specific role(s) of wide spread antisense transcription in recent years, the functional significance of NATs and their physiological regulation remains poorly understood. However, the significant presence of NATs in the central nervous system suggests their potential role in brain function.
The role of NATs in establishing memory formation has been suggested in Lymnaea where axonal transport of NAT, described as antiNOS-2 RNA, is regulated by classical conditioning. AntiNOS2 RNA negatively regulates the neurotransmitter nitric oxide (NO), a key transcript that plays an important role in the early stages of learning and memory formation (Korneev et al., 2013). It is not clear whether classical conditioning will lead to a net enhancement of antiNOS2 expression in the cell body and processes of CGC neuron or a selective increase in expression in the periphery.

To investigate physiological mechanisms that regulate expression and subcellular distribution of NATs, we have explored the expression of a NAT transcribed against the mRNA encoding the peptide neurotransmitter sensorin and its physiological regulation in the sensory neurons (SN) of the marine mollusk Aplysia californica. For more than 50 years Aplysia has provided fundamental insights into the basic organization of neuronal functions. Aplysia's nervous system has large neurons, many of them can be uniquely identified and are associated with specific behaviors. These neurons can be isolated and cultured in vitro and they form circuits, which can be investigated at the molecular and cellular detail.

The cell-specific neuropeptide, sensorin (SRN), is expressed exclusively in SNs and transported to distal neurites (Brunet et al., 1991). However, the distribution of sensorin transcripts in the SN cell bodies change when it is co-cultured with a motor neuron (Hu et al., 2002, 2003). It has been shown that formation and stabilization of sensory neuron (SN)-motor neuron (MN) synapses are regulated upon the release of sensorin peptide from 
SNs (Hu et al., 2004). In vitro, SNs uniquely make synapses with their in vivo target motor neurons but not with their non-target motor neurons, providing an excellent model system to specifically study the effects of specific mRNAs in synapse formation and stabilization (Kandel, 2001).

Figure 1D depicts the schematic diagram showing our strategy to study NAT-SRN in sensory neurons. Our analysis of gene expression using qPCR, northern blotting and single neuron quantitative PCR (qPCR) and fluorescent in situ hybridization (FISH) analyses have confirmed the expression of sense (SRN) and antisense RNAs (NAT-SRN) of neuropeptide sensorin in SNs of Aplysia gill withdrawal reflex. We then examined whether expression of NAT-SRN transcripts is regulated in SNs during aging and in response to forskolin, an activator of CREB (Seternes et al., 1999). We find that the expression levels and sub-cellular distribution of NAT-SRN are differentially altered during aging and neuronal activity.

\section{MATERIALS AND METHODS ETHICS STATEMENT}

The Institutional Biosafety Committee of The Scripps Research Institute (TSRI) has approved all of the experimental protocols (IBC Protocol 2010-019R1) described in this manuscript. Ethical approvals are not required for the research using invertebrate animals, such as the marine snail Aplysia.

\section{ANIMALS, ISOLATION OF APLYSIA NEURONS, AND CULTURE}

Aplysia californica maintained under standard conditions (temperature, salinity, $\mathrm{pH}$, food) at the National Aplysia Resource Facility (University of Miami Rosenstiel School of Medicine, Florida, USA) were used in the experiment. In our study, we used animals that correspond to two age groups (2 and 9 months old). Upon arrival in the laboratory, animals were kept in an aquarium at $16^{\circ} \mathrm{C}$, under 12:12 light-dark conditions and was used for experiments within 2-3 days of arrival. Isolation of sensory neurons and culture were done as described earlier (Montarolo et al., 1986). Micro-dissection of cell body and processes were carried out as described earlier (Moccia et al., 2003; Moroz et al., 2006).

\section{NORTHERN BLOT}

A 415-bp DNA fragment corresponding to exons 1 and 2 of sensorin mRNA was cloned into TOPO Vector with dual promoters T7 and SP6 (Invitrogen, Cat. Number K4600-01) and then in vitro transcribed to prepare DIG labeled sense and antisense riboprobes using the DIG RNA Labeling Kit (SP6/T7) (Roche Diagnostics). The molecular sizes of riboprobes were confirmed by gel electrophoresis. Electrophoresis and transfer of the RNA was performed using DIG Northern Starter Kit (Roche Applied Science) and followed manufacturer's protocols. Briefly, 5 and $10 \mu \mathrm{g}$ of total RNA from Aplysia was run on $1 \%$ agarose gel, transferred to a positive nitrocellulose membrane following hybridization with strand-specific riboprobes at $68^{\circ} \mathrm{C}$ overnight with ExpressHyb.

\section{RNA EXTRACTION AND REVERSE TRANSCRIPTION}

Total RNA was extracted from the Aplysia CNS using the standard RNA Trizol extraction method and dissolved in nuclease-free water. For the preparation of RNA from SNs, cell body and neurites were separated by manual micro-dissection followed by RNA extraction using Trizol. RNAs were amplified once using MessageAmp ${ }^{\mathrm{TM}}$ II aRNA Amplification Kit following manufacturer's instructions. For the CNS samples $1 \mu \mathrm{g}$ of the total RNA, and for the cell body and neurites, $500 \mathrm{ng}$ of RNAs were used for reverse transcription using qScript cDNA synthesis mix.

\section{QUANTITATIVE REAL-TIME PCR}

Primer pairs for SRN, NAT-SRN, and ApKHC1 and ApKLC2 were designed using the Primer3 program (http://bioinfo.ut.ee/ primer3-0.4.0/) based on cDNA and genome sequences listed in UCSC genome browser database (http://genome.ucsc.edu/) (Figure 1A). While designing, primers steps are taken to avoid the amplification of multiple targets. Antisense primers were designed based on the exon boundaries of sense transcript to avoid potential amplification of the sense transcripts in the antisense detection samples. $2 \mu \mathrm{l}$ of the cDNA synthesized was used for qPCR following the protocol described earlier (Kadakkuzha et al., 2013; Akhmedov et al., 2014). Briefly, $10 \mu l$ reactions contained $2 \mu \mathrm{l}$ of cDNA, $8 \mu \mathrm{l}$ of a $\mathrm{qPCR}$ master mix containing $2 \mu \mathrm{l}$ of $\mathrm{H}_{2} \mathrm{O}, 5 \mu \mathrm{l}$ of $2 \mathrm{X}$ SYBR Green master mix, and $1.0 \mu \mathrm{l}$ of $10 \mu \mathrm{M}$ (each) forward and reverse primer. The reaction was carried out in a 7900HT Fast Real-Time PCR System (Applied Biosystems Carlsbad, CA) under the following conditions: $95^{\circ} \mathrm{C}$ for $10 \mathrm{~min}$, followed by 40 cycles of $95^{\circ} \mathrm{C}$ for $15 \mathrm{~s}, 60^{\circ} \mathrm{C}$ for $1 \mathrm{~min}$. Five biological replicates and four technical replicates for each biological replicate were used in the qPCR. Quantification of the target transcripts was normalized to the Aplysia18S reference gene using the Pfaffl method (Pfaffl, 2001). Data are shown as mean \pm s.e.m. Statistical analysis was performed using Prism (GraphPad Software). Student's $t$-test or ANOVA followed by Bonferroni's test were used as appropriate where ${ }^{*} P$-value $<0.05$, ${ }^{* *} P$-value $<0.01,{ }^{* * *} P$-value $<0.001$.

\section{PREPARATION OF DIG LABELED RIBO PROBES}

DIG labeled sense and antisense ribo probes for in situ hybridization probes were prepared by in vitro transcription of cDNA templates by using SP6 or T7 RNA polymerase. $415 \mathrm{nt}$ long coding region of sensorin was prepared by PCR using Aplysia abdominal ganglion cDNA as a template and sensorin specific PCR primers and ligated to pCRII-TOPO Vector with dual promoters T7 and SP6 (Invitrogen, Cat. Number K4600-01). The Vector with the sensorin DNA was linearized with EcoR V (New England Biolab) for transcription using SP6 RNA polymerase to generate antisense probes and the corresponding sense probes were produced by linearizing with BamH I (New England Biolab) and transcribing with T7 RNA polymerase. A small aliquot $(2 \mu \mathrm{l})$ was run on $1.5 \%$ agarose gel to confirm the integrity of RNA probes.

\section{FLUORESCENT IN SITU HYBRIDIZATION (FISH) AND IMAGING ANALYSIS}

DIG labeled RNA probes were prepared and in situ hybridization analysis of sensory neurons were carried out as described in (Puthanveettil et al., 2013). Images were acquired using a Zeiss LSM 780 confocal microscope system with 10X/63X objective; only projection images are shown. Mean fluorescence intensities 


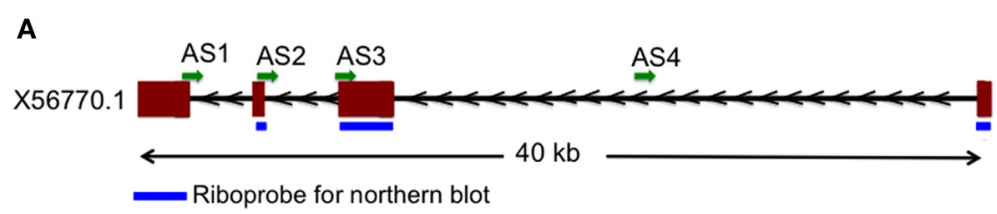

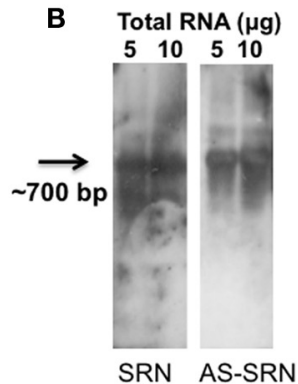

C

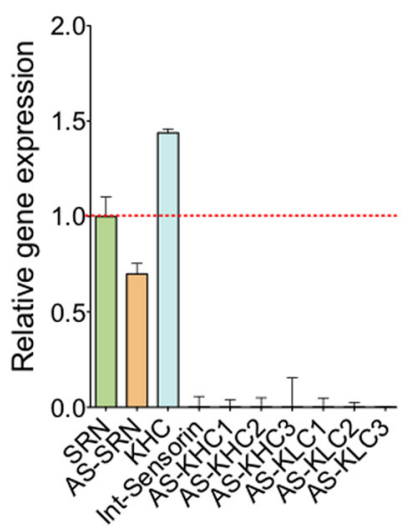

FIGURE 1 | Identification of natural antisense transcripts of neuropeptide sensorin. Arrows indicate the direction of transcription. (A) The psc1 gene (EST X56770) coding for sensorin mRNA has four exons spanning $\sim 40 \mathrm{~KB}$ on the scaffold 926 of Aplysia. AS1-4 represents the locations of qPCR amplicons that are used to detect AS transcript. (B) Northern blot detection of SRN and NAT-SRN from the total RNA of Aplysia CNS using strand specific ribo-probes. (C) qPCR analysis of SRN
D
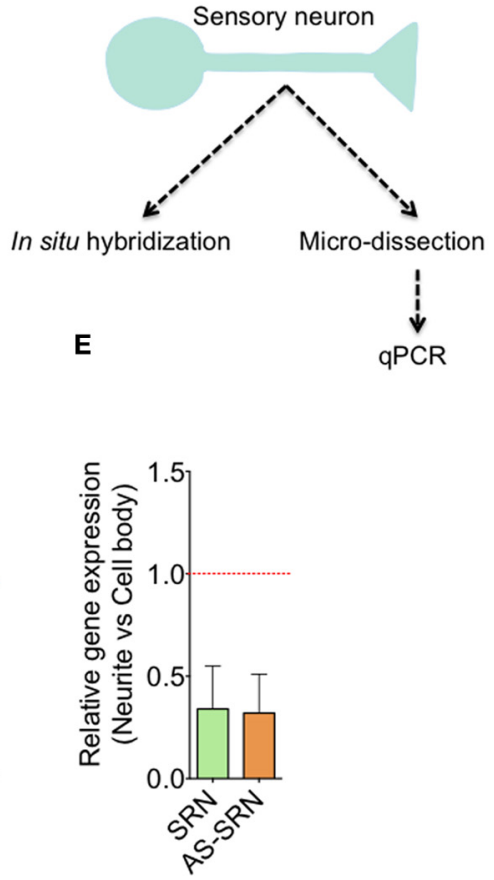

and NAT-SRN in RNA samples from Aplysia CNS. Primers that can amplify antisense direction of kinesin heavy chain, kinesin light chain and the primers to amplify intronic region of sensorin were used as negative controls. (D) Schematic diagram showing our strategy to study NAT-SRN in sensory neurons (E) qPCR analysis of SRN and NAT-SRN in the cell body and neurites of sensory neurons. Data was normalized to 18s rRNA levels. Error bars are SEM. were measured using NIH IMAGE J and normalized intensities were calculated using the following equation:

Normalized intensity $=$ Mean FISH intensity $($ SRN or NATSRN) - Mean background signal.

Distributions of $\beta$-tubulin protein in both cell body and neuritis were measured to identify any non-specific changes in protein expression associated with aging or forskolin treatment.

\section{RESULTS}

\section{DETECTION OF SENSORIN SENSE AND ANTISENSE TRANSCRIPTS IN APLYSIA CNS}

Existence of sensorin antisense transcript (NAT-SRN) was previously suspected while performing in situ hybridization analysis of expression of sensorin in sensory neurons using fluorescently labeled sense (S) and antisense (AS) riboprobes (unpublished data). Sensorin mRNA (SRN) is transcribed from pscl gene on the reverse strand of scaffold 926 of Aplysia genome (available through UCSC genome browser), spanning $40 \mathrm{~KB}$ long region and contains four exons (Figure 1A). In order to further characterize the sense (S) and the NAT at the pscl locus, we searched for the presence of the NATs by northern blot, qPCR and in situ hybridization. Using DIG labeled ribo probes to detect SRN and NAT-SRN, we first analyzed total RNAs from the central nervous system (CNS) by northern hybridization analysis. Figure 1B show hybridization signals for SRN and NAT-SRN corresponding to $\sim 700$ nucleotides suggesting that NAT-SRN transcripts are expressed in the CNS.

Using specific primers (Supplementary Table 1) that detect SRN and NAT-SRN CDNA, we then confirmed the presence of SRN and NAT-SRN by qPCR. Primers designed to amplify the intronic regions of the SRN gene were used as negative controls and to make sure that the products were not generated from 
genomic DNA. From multiple primers that we used we selected AS2 (Figure 1A) for further qPCR detection of NAT-SRN. Primers to detect putative NAT of Aplysia kinesin heavy chain (KHC1) and KLC2 (Supplementary Table 1) were used as additional controls in qPCRs (Figure 1C). Data was normalized to $18 \mathrm{~S}$ rRNA levels. qPCR results showed that SRN was expressed at $\sim 30 \%$ higher levels when compared to NAT-SRN levels in CNS $(p=0.0235$, ANOVA, Figure 1C).

\section{NAT-SRN IS EXPRESSED IN PROCESSES OF SENSORY NEURONS}

Previous studies have shown that release of sensorin from the SN is required for both synapse formation and long-term facilitation (LTF) of SN to MN connections. Localization of sensorin is modulated by the formation of synapses between $\mathrm{SN}$ and its target motor neurons (Lyles et al., 2006). We examined the distribution of both SRN and NAT-SRN isolated sensory neurons by qPCR. Analysis of RNAs isolated from micro dissected cell bodies and processes (Figure 1C) showed that both SRN and NAT-SRN transcripts are present in the neurites however, their levels in the cell bodies are much higher than that of neurites $[\sim 2$-fold decrease in expression of SRN and NAT-SRN transcripts in the neurites compared to cell body, Figure 1E, Student's $t$-test; $p=0.043$ (SRN), $p=0.027$ (NAT-SRN)]. As a control we examined expression of Aplysia KHC1 transcripts in SN neurites. Specific primers for KHC1 were unable to detect expression of antisense or sense transcripts in SN neurites.

\section{EXPRESSION OF NAT-SRN CHANGE DURING AGING}

We next examined whether expression of NAT-SRN is physiologically regulated. We first studied whether aging is associated with a change in the distribution of NAT-SRN and looked at the distribution of SRN and NAT-SRN in sensory neurons cultured from 3 to 9 month old Aplysia. From FISH analysis it is evident that both SRN and NAT-SRN are present in the cell body of SNs from young and old animals (Figure 2A). The level of SRN transcript did not change notably in the cell bodies of neurons from young and old animals (Figure $2 \mathbf{B}, N=4$, Students' $t$-test, $p=0.8854$ ) indicating that SRN transcript level in the cell body is not affected by aging. However, we observed a significant increase in the distribution of NAT-SRN in the cell body of SNs from old animals (Figure 2C, \% increase: $25 \pm$ $8, N=4$, Students' $t$-test, $p=0.0362$ ). As an endogenous control we used the expression of $\beta$-tubulin and found that the $\beta$-tubulin protein levels in cell bodies of young and old neurons did not change significantly $(N=4$, Students' $t$-test, $p=0.7504)$ (Figure 2D).

We next analyzed the distribution of NAT-SRN in the processes of SNs cultured from young and old animals by FISH analysis and found moderate levels of SRN and NAT-SRN in the neurites of young and old animals (Figure 3A). Comparison of the mean intensities of FISH signals from young and old SRN and NATSRN after background signal subtraction suggested no change in the distribution of SRN transcript in the neurites of SNs from the young and old animals; an observation similar to what we found in the cell bodies described earlier (Figure 3B, $N=4$, Students' $t$ test, $p=0.0864$ ). Also, we did not observe any significant change in the level of NAT-SRN in the neurites of SNs from old animals when compared to young animals (Figure 3C, $N=4$, Students'

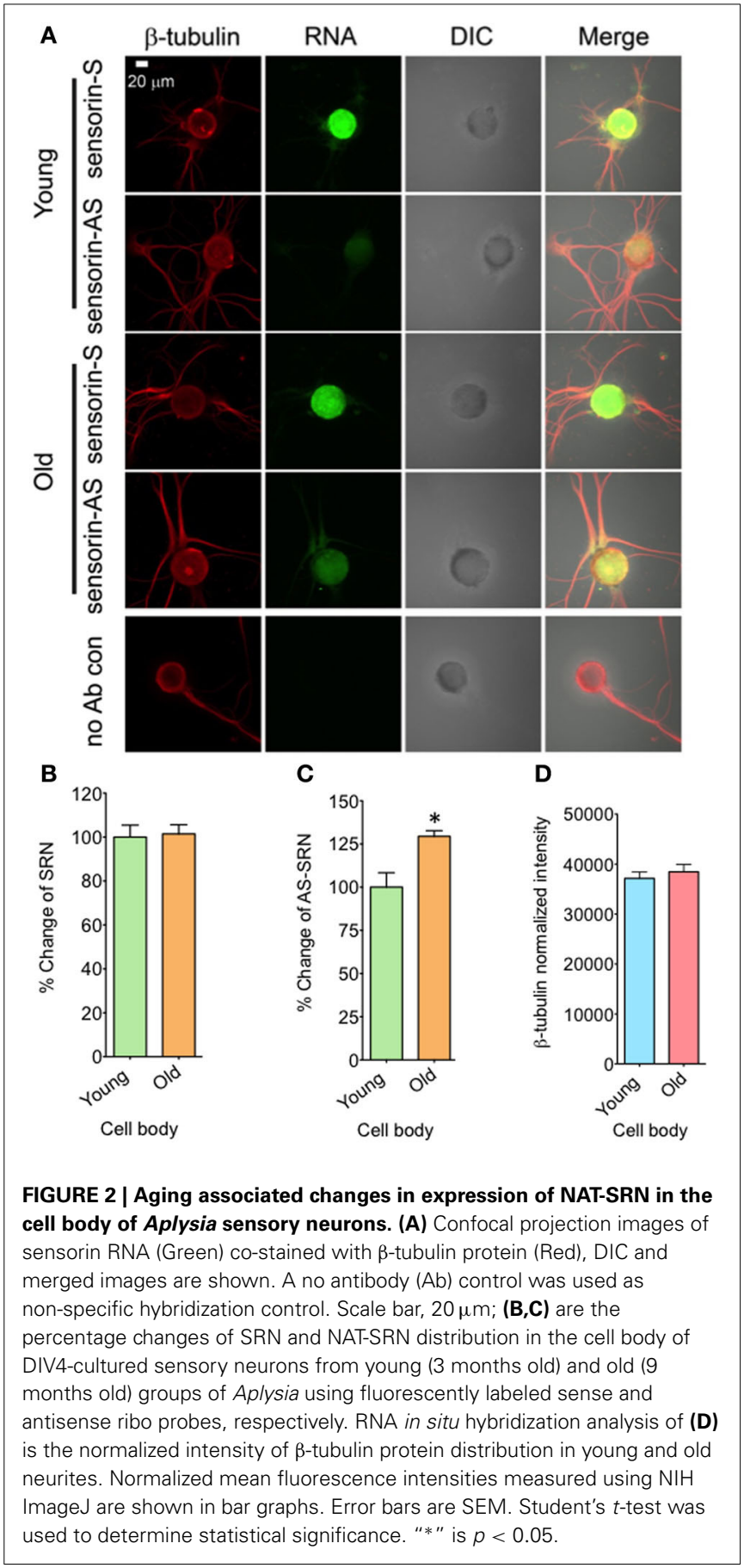

$t$-test, $p=0.9403)$. The endogenous control $\beta$-tubulin protein levels in processes of young and old neurons did not change significantly $(N=4$, Students' $t$-test, $p=0.7701)$ (Figure 3D). These results suggest that the expression and localization of NAT-SRN transcripts are regulated during aging.

\section{FORSKOLIN EXPOSURE ENHANCE TRANSCRIPTION OF NAT-SRN IN BOTH CELL BODIES AND NEURITES}

We next studied whether NAT-SRN levels could be regulated by forskolin, an activator of cAMP-CREB signaling. SN 


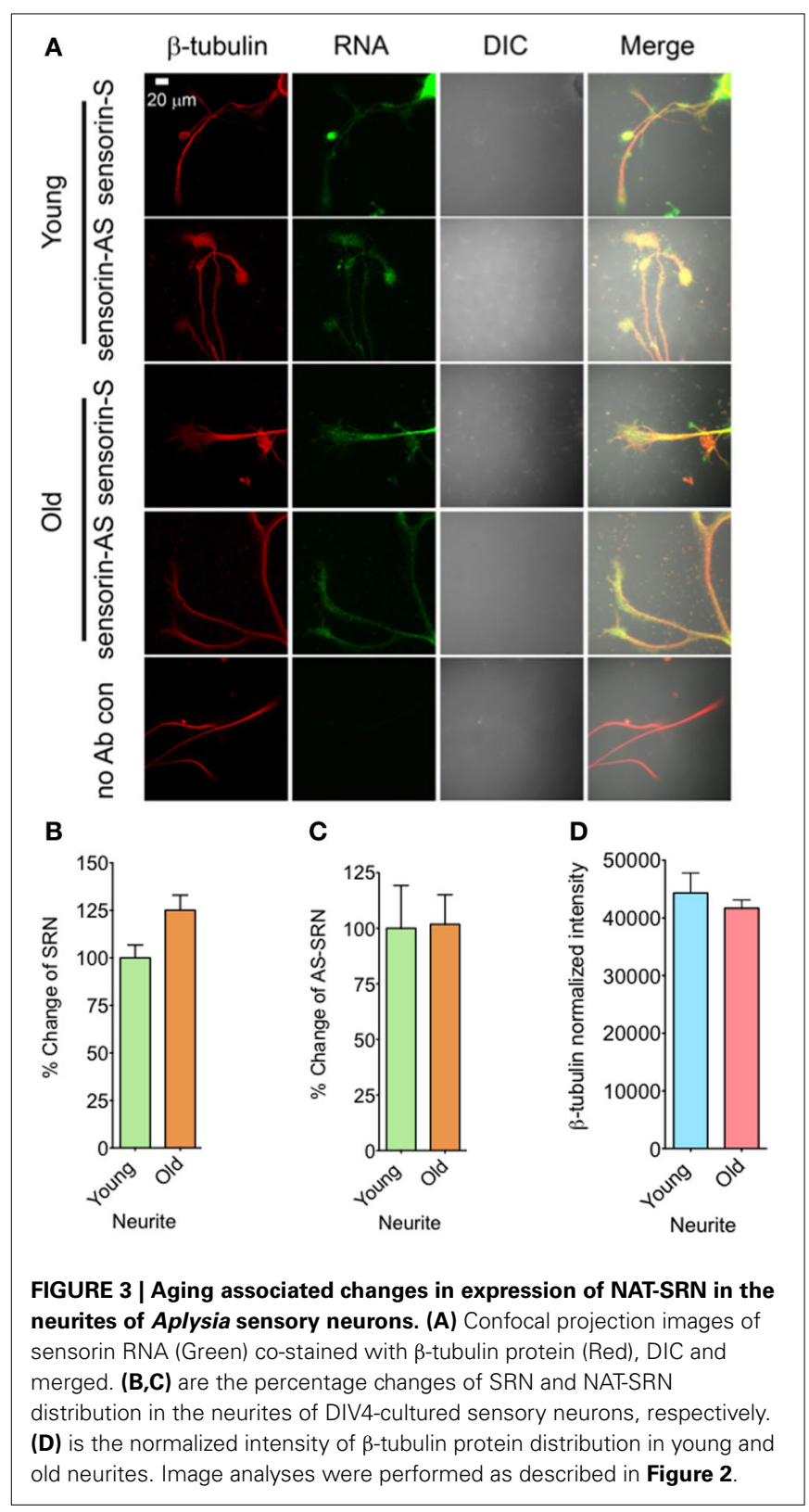

cultures ( $4 \mathrm{DIV})$ were treated with $50 \mu \mathrm{M}$ forskolin for $30 \mathrm{~min}$ (Puthanveettil et al., 2008) and fixed for FISH analysis (Figure 4A). Vehicle treated SNs were used as controls. Analysis of mean fluorescence intensities of expression of SRN and NATSRN suggest that forskolin treatment induced 25\% increase in the expression level of SRN in the cell body (Figure 4B) but no change in NAT-SRN level (Student's $t$-test; $p=0.0007)$. Interestingly, there was no change in the level of SRN in the neurites of SNs treated with forskolin within 30 min of treatment but the NAT-SRN level was increased by $50 \%$ after forskolin treatment in the neurites (Figures 4D,E; Student's $t$-test; $p=0.001$ ). $\beta$-tubulin protein levels in the cell body (Figure 4C) and processes (Figure 4F) of control and forskolin treated neurons did not change significantly $(N=4$, Students' $t$-test, $p=0.7225$ and 0.6712 , respectively).

\section{DISCUSSION}

Advances in sequencing methodologies have led to sequencing of several genomes and transcriptomes shedding light on the noncoding component of the genome. These large-scale sequencing studies have resulted in cataloging of thousands of non-coding RNAs in a variety of organisms (Katayama et al., 2005; Li et al., 2013). We have now begun to understand the functional relevance of transcription of non-coding RNAs and physiological mechanisms that regulate transcription of non-coding RNAs. Non-coding RNAs include miRNAs, piRNAs, tRNAs, rRNAs, snRNAs, large non-coding RNAs, and natural antisense RNAs. NAT are intriguing because these RNAs have transcripts complementary to other RNA transcripts. Several regulatory roles for NATs have been suggested including RNA interference, genomic imprinting, and alternate splicing (Zhang et al., 2007; Faghihi and Wahlestedt, 2009; Werner, 2013; Wight and Werner, 2013).

Recent studies have demonstrated that NATs could downregulate expression of its complementary transcripts. For example, in the rice plant, NATs regulate expression of a protein important for phosphate homeostasis (Jabnoune et al., 2013). Similarly NAT of interleukin (IL) 1 beta) (Lu et al., 2013), iNOS (inducible nitric oxide synthase) (Yoshigai et al., 2013), and ubiquitin cterminal hydrolase (uch) (Carrieri et al., 2012), Huntington's disease (Chung et al., 2011) suppress expression of their complementary transcripts. Importantly, inhibition of expression of NATs has resulted in upregulation of gene specific transcription (Modarresi et al., 2012). Recently, (Velmeshev et al., 2013) showed that $40 \%$ of loci previously implicated in autism spectrum disorders express NATs. These NATs are expressed in specific brain regions.

We are only beginning to understand the physiological processes and mechanisms that regulate expression of NATs. In plants, drought alters expression of NATs (Lembke et al., 2012). Similarly during beta amyloid induced apoptosis, NAT of Rad18 gene become upregulated (Parenti et al., 2007). Also, during corticogenesis in mouse brain the expression of NAT of Nrgn and CamK2n1 are regulated (Ling et al., 2011). Additionally, it has been shown that sense-antisense transcript pairs are present in synaptoneurosomes (Smalheiser et al., 2008).

Despite these elegant studies, we still do not know whether and how NATs are regulated in specific neural circuitries. To address this we used well-characterized neural circuitry in Aplysia, the sensory-motor neurons of gill withdrawal reflex (Kandel, 2001) and studied potential NAT of neuropeptide sensorin (NAT-SRN) in sensory neurons. Neuropeptide sensorin is expressed in presynaptic sensory neurons and is important for LTF of sensory to motor neuron synapses (Brunet et al., 1991; Schacher et al., 1999). Sensorin RNA is transported to synapses (Schacher et al., 1999; Moccia et al., 2003) and translated in response to repeated 5-HT stimulation (Wang et al., 2009). To search for putative NAT-SRN, we first analyzed Aplysia genome and designed primers to prepare sense and antisense probes for northern analysis. We find that NAT-SRN is expressed in Aplysia CNS and that it probably has a similar molecular weight as compared to complementary sense transcript. We next confirmed the expression of NAT-SRN by qPCR analysis of CNS and micro-dissected cell body and processes of individual SNs. Having confirmed the expression 

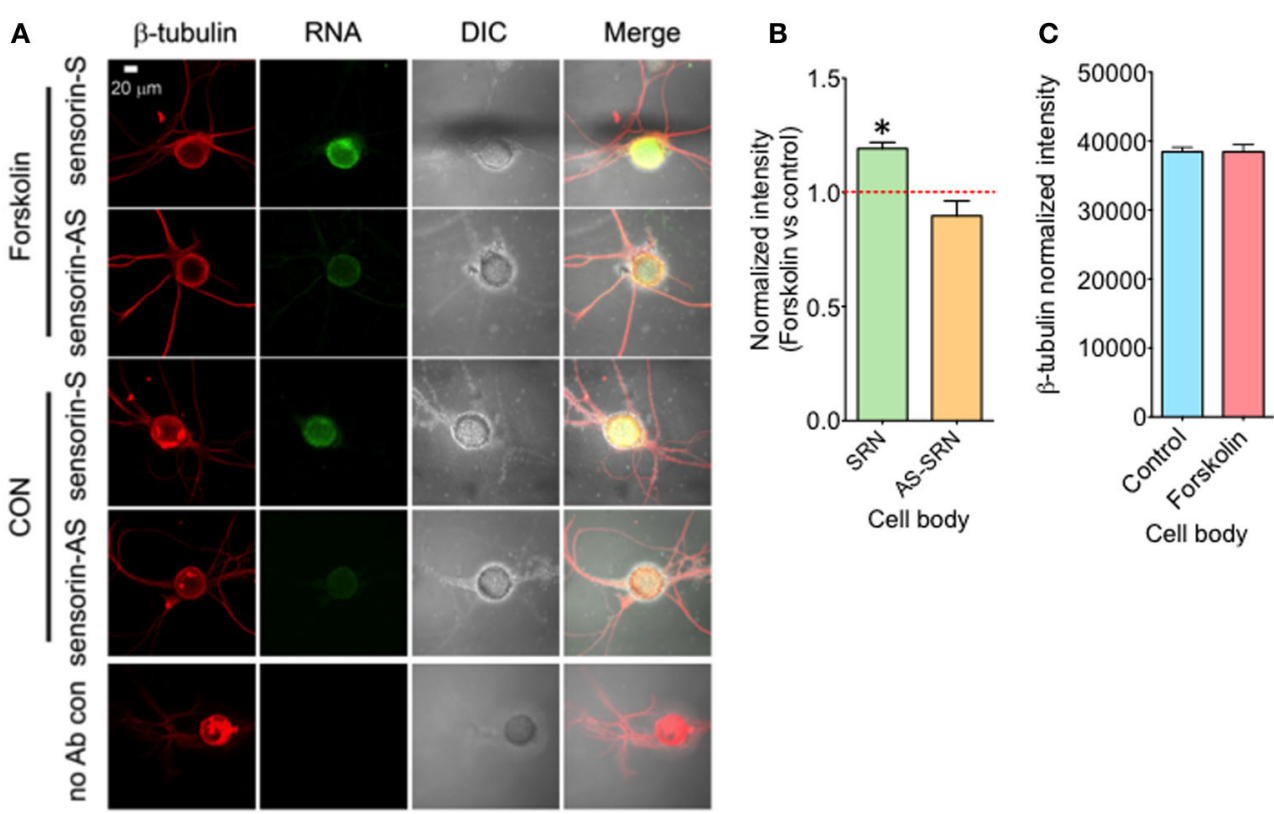

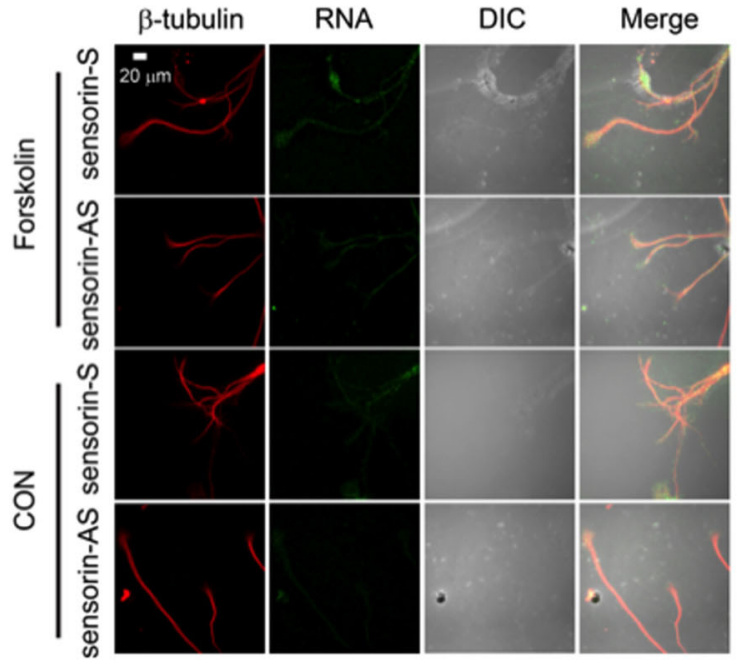

$\mathbf{E}$

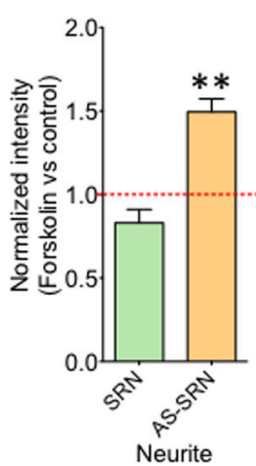

$\mathbf{F}$

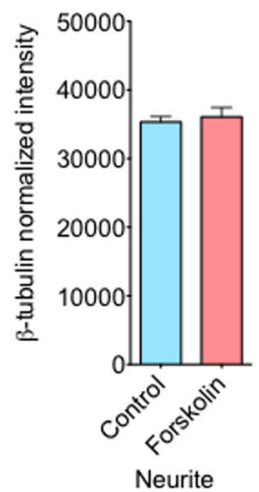

FIGURE 4 | Forskolin exposure enhances expression of NAT-SRN in

Aplysia sensory neurons. Sensory neurons of mature Aplysia were cultured in vitro and were treated with forskolin $(50 \mu \mathrm{M}, 30 \mathrm{~min})$ on DIV 4. (A) Confocal projection images of FISH analysis of sensorin RNA (SRN) (Green) and (D) that of AS-sensorin RNA (NAT-SRN) (Green) co-stained with $\beta$-tubulin protein (Red), as well as DIC images are shown. A no antibody control was used as non-specific hybridization control. Scale bar, $20 \mu \mathrm{m}$. (B,E) show the change in expression levels of SRN and NAT-SRN in forskolin treated neurons when compared with vehicle treated control neurons in cell body and neuritis, respectively. $(\mathbf{C}, \mathbf{F})$ are the normalized intensity of $\beta$-tubulin protein levels in control and forskolin treated cell body and neuritis, respectively. Normalized mean fluorescence intensities measured using NIH ImageJ are shown in bar graphs. Error bars are SEM. Student's t-test was used to determine statistical significance. "*" is $p<0.05, "$ "**" is $p<0.01$. of NAT-SRN in SNs, we then asked two questions: (a) whether NAT-SRN is physiologically regulated and (b) whether NATSRN expression could be regulated by cellular activities that elicit specific signaling pathways leading to memory storage.

To understand physiological regulation of NAT-SRN, we first determined whether its expression changes during aging and whether aging causes changes in subcellular distribution of NATSRN. Importantly, aging associated changes in specific NATs are poorly understood. We studied two age groups, young and old Aplysia. Our FISH analyses suggest that the amount of NATSRN increases in cell body of old neurons when compared to corresponding sense transcripts. However, the NAT-SRN level is decreased in neurites of sensory neurons cultured from old animals.

To determine whether specific cellular activities might regulate expression of NAT-SRN, we measured changes in expression 
of NAT-SRN in response to forskolin, an activator of cAMPPKA-CREB pathway important for long-term memory storage (Kandel, 2001). Recently (Korneev et al., 2013) have shown that classical conditioning of Lymnaea changes in the expression of NAT of nitric oxide synthase. Consistent with the idea that NATs could be physiologically regulated, we find that immediately after forskolin treatment, there is an increase in SRN transcripts in the cell body. However, forskolin did not cause an increase in the expression of NAT-SRN in the cell body. Interestingly we find that forskolin exposure resulted in a significant increase in NAT-SRN transcripts in the neurites.

The differential subcellular localization of sense and NATSRN during aging and in response to forskolin treatment suggest that there might be specific mechanisms that mediate differential expression and transport of SRN and NAT-SRN transcripts. We consider three possible mechanisms: (a) regulation of transcription of NAT-SRN or (b) degradation of NAT-SRN in specific compartments, or (c) changes in axonal transport of NAT-SRN to neurites. It has been shown that expression of NATs could be regulated by epigenetic mechanisms (Conley and Jordan, 2012). We find that exposure to forskolin cause a rapid increase in sense transcripts in cell body and increase in NAT-SRN in neurites. Our observation that forskolin did not cause upregulation of NAT-SRN in the cell body suggests the possibility that transcription of NAT-SRN is not regulated by CREB. We have previously shown that (Puthanveettil et al., 2008) forskolin treatment cause a rapid increase in kinesin mRNA levels, the molecular motor that mediates axonal transport, in sensory neurons. However, it is yet to be determined whether NAT-SRN is transported by kinesin and whether increase in NAT-ARN in SN neurites correlate with enhanced expression of kinesin mRNAs.

In summary, we have identified expression of natural antisense RNA of sensorin, an important neuropeptide involved in memory storage. We find that its subcellular localization in sensory neurons is differentially regulated during aging and in response to activation of cAMP-PKA-CREB pathway. We now provide evidence that NATs could be differentially regulated in different sub-cellular compartments. Further studies are required to delineate mechanisms and understand physiological implications of such regulation.

\section{ACKNOWLEDGMENTS}

We sincerely thank the Whitehall Foundation, NIMH grant 1 R21MH096258-01A1 and The Scripps Research Institute for their funding support which helped us carry out this research.

\section{SUPPLEMENTARY MATERIAL}

The Supplementary Material for this article can be found online at: http://www.frontiersin.org/journal/10.3389/fgene.2014. 00084/abstract

\section{REFERENCES}

Akhmedov, K., Kadakkuzha, B. M., and Puthanveettil, S. V. (2014). Aplysia Ganglia preparation for electrophysiological and molecular analyses of single neurons. J. Vis. Exp. e51075. doi: 10.3791/51075

Brunet, J. F., Shapiro, E., Foster, S. A., Kandel, E. R., and Iino, Y. (1991). Identification of a peptide specific for aplysia sensory neurons by Pcr-Based differential screening. Science 252, 856-859. doi: 10.1126/science.1840700
Carrieri, C., Cimatti, L., Biagioli, M., Beugnet, A., Zucchelli, S., Fedele, S., et al. (2012). Long non-coding antisense RNA controls Uchll translation through an embedded SINEB2 repeat. Nature 491, 454-457. doi: 10.1038/nature11508

Chung, D. W., Rudnicki, D. D., Yu, L., and Margolis, R. L. (2011). A natural antisense transcript at the Huntington's disease repeat locus regulates HTT expression. Hum. Mol. Genet. 20, 3467-3477. doi: 10.1093/hmg/ddr263

Conley, A. B., and Jordan, I. K. (2012). Epigenetic regulation of human cis-natural antisense transcripts. Nucleic Acids Res. 40, 1438-1445. doi: 10.1093/nar/gkr1010

Faghihi, M. A., and Wahlestedt, C. (2009). Regulatory roles of natural antisense transcripts. Nat. Rev. Mol. Cell Biol. 10, 637-643. doi: 10.1038/nrm2738

Hu, J. Y., Goldman, J., Wu, F., and Schacher, S. (2004). Target-dependent release of a presynaptic neuropeptide regulates the formation and maturation of specific synapses in Aplysia. J. Neurosci. 24, 9933-9943. doi: 10.1523/JNEUROSCI.332904.2004

Hu, J. Y., Meng, X., and Schacher, S. (2002). Target interaction regulates distribution and stability of specific mRNAs. J. Neurosci. 22, 2669-2678. doi: 20026259

Hu, J. Y., Meng, X., and Schacher, S. (2003). Redistribution of syntaxin mRNA in neuronal cell bodies regulates protein expression and transport during synapse formation and long-term synaptic plasticity. J. Neurosci. 23, 1804-1815.

Jabnoune, M., Secco, D., Lecampion, C., Robaglia, C., Shu, Q., and Poirier, Y. (2013). A Rice cis-natural antisense RNA acts as a translational enhancer for its cognate mRNA and contributes to phosphate homeostasis and plant fitness. Plant Cell 25, 4166-4182. doi: 10.1105/tpc.113.116251

Kadakkuzha, B. M., Akhmedov, K., Capo, T. R., Carvalloza, A. C., Fallahi, M., and Puthanveettil, S. V. (2013). Age-associated bidirectional modulation of gene expression in single identified R15 neuron of Aplysia. BMC Genomics 14:880. doi: $10.1186 / 1471-2164-14-880$

Kandel, E. R. (2001). The molecular biology of memory storage: a dialogue between genes and synapses. Science 294, 1030-1038. doi: 10.1126/science.1067020

Katayama, S., Tomaru, Y., Kasukawa, T., Waki, K., Nakanishi, M., Nakamura, M., et al. (2005). Antisense transcription in the mammalian transcriptome. Science 309, 1564-1566. doi: 10.1126/science.1112009

Korneev, S. A., Kemenes, I., Bettini, N. L., Kemenes, G., Staras, K., Benjamin, P. R., et al. (2013). Axonal trafficking of an antisense RNA transcribed from a pseudogene is regulated by classical conditioning. Sci. Rep. 3, 1027. doi: $10.1038 /$ srep01027

Lembke, C. G., Nishiyama, M. Y. Jr., Sato, P. M., De Andrade, R. F., and Souza, G. M. (2012). Identification of sense and antisense transcripts regulated by drought in sugarcane. Plant Mol. Biol. 79, 461-477. doi: 10.1007/s11103-012-9922-1

Li, J., Xuan, Z., and Liu, C. (2013). Long non-coding RNAs and complex human diseases. Int. J. Mol. Sci. 14, 18790-18808. doi: 10.3390/ijms140918790

Ling, K. H., Hewitt, C. A., Beissbarth, T., Hyde, L., Cheah, P. S., Smyth, G. K., et al. (2011). Spatiotemporal regulation of multiple overlapping sense and novel natural antisense transcripts at the Nrgn and Camk2n1 gene loci during mouse cerebral corticogenesis. Cereb. Cortex 21, 683-697. doi: 10.1093/cercor/bhq141

Lu, J., Wu, X., Hong, M., Tobias, P., and Han, J. (2013). A potential suppressive effect of natural antisense IL-1beta RNA on lipopolysaccharide-induced IL1beta expression. J. Immunol. 190, 6570-6578. doi: 10.4049/jimmunol.1102487

Lyles, V., Zhao, Y., and Martin, K. C. (2006). Synapse formation and mRNA localization in cultured Aplysia neurons. Neuron 49, 349-356. doi: 10.1016/j.neuron.2005.12.029

Moccia, R., Chen, D., Lyles, V., Kapuya, E., E, Y., Kalachikov, S., et al. (2003). An unbiased cDNA library prepared from isolated Aplysia sensory neuron processes is enriched for cytoskeletal and translational mRNAs. J. Neurosci. 23, 9409-9417.

Modarresi, F., Faghihi, M. A., Lopez-Toledano, M. A., Fatemi, R. P., Magistri, M., Brothers, S. P., et al. (2012). Inhibition of natural antisense transcripts in vivo results in gene-specific transcriptional upregulation. Nat. Biotechnol. 30, 453-459. doi: 10.1038/nbt.2158

Modarresi, F., Faghihi, M. A., Patel, N. S., Sahagan, B. G., Wahlestedt, C., and Lopez-Toledano, M. A. (2011). Knockdown of BACE1-AS nonprotein-coding transcript modulates beta-amyloid-related hippocampal neurogenesis. Int. J. Alzheimers Dis. 2011, 929042. doi: 10.4061/2011/929042

Montarolo, P. G., Goelet, P., Castellucci, V. F., Morgan, J., Kandel, E. R., and Schacher, S. (1986). A critical period for macromolecular synthesis in long-term heterosynaptic facilitation in Aplysia. Science 234, 1249-1254. doi: 10.1126/science. 3775383 
Moroz, L. L., Edwards, J. R., Puthanveettil, S. V., Kohn, A. B., Ha, T., Heyland, A., et al. (2006). Neuronal transcriptome of Aplysia: neuronal compartments and circuitry. Cell 127, 1453-1467. doi: 10.1016/j.cell.2006.09.052

Parenti, R., Paratore, S., Torrisi, A., and Cavallaro, S. (2007). A natural antisense transcript against Rad18, specifically expressed in neurons and upregulated during beta-amyloid-induced apoptosis. Eur. J. Neurosci. 26, 2444-2457. doi: 10.1111/j.1460-9568.2007.05864.x

Pelechano, V., and Steinmetz, L. M. (2013). Gene regulation by antisense transcription. Nat. Rev. Genet. 14, 880-893. doi: 10.1038/nrg3594

Pfaffl, M. W. (2001). A new mathematical model for relative quantification in realtime RT-PCR. Nucleic Acids Res. 29, e45. doi: 10.1093/nar/29.9.e45

Puthanveettil, S. V., Antonov, I., Kalachikov, S., Rajasethupathy, P., Choi, Y. B., Kohn, A. B., et al. (2013). A strategy to capture and characterize the synaptic transcriptome. Proc. Natl. Acad. Sci. U.S.A. 110, 7464-7469. doi: 10.1073/pnas.1304422110

Puthanveettil, S. V., Monje, F. J., Miniaci, M. C., Choi, Y. B., Karl, K. A., Khandros, E., et al. (2008). A new component in synaptic plasticity: upregulation of kinesin in the neurons of the gill-withdrawal reflex. Cell 135, 960-973. doi: 10.1016/j.cell.2008.11.003

Schacher, S., Wu, F., Panyko, J. D., Sun, Z. Y., and Wang, D. (1999). Expression and branch-specific export of mRNA are regulated by synapse formation and interaction with specific postsynaptic targets. J. Neurosci. 19, 6338-6347.

Seternes, O. M., Johansen, B., and Moens, U. (1999). A dominant role for the Raf-MEK pathway in forskolin, 12-O-tetradecanoyl-phorbol acetate, and platelet-derived growth factor-induced CREB (cAMP-responsive elementbinding protein) activation, uncoupled from serine 133 phosphorylation in NIH 3T3 cells. Mol. Endocrinol. 13, 1071-1083. doi: 10.1210/mend.13.7.0293

Smalheiser, N. R., Lugli, G., Torvik, V. I., Mise, N., Ikeda, R., and Abe, K. (2008). Natural antisense transcripts are co-expressed with sense mRNAs in synaptoneurosomes of adult mouse forebrain. Neurosci. Res. 62, 236-239. doi: 10.1016/j.neures.2008.08.010

Velmeshev, D., Magistri, M., and Faghihi, M. A. (2013). Expression of nonprotein-coding antisense RNAs in genomic regions related to autism spectrum disorders. Mol. Autism. 4, 32. doi: 10.1186/2040-2392-4-32
Wang, D. O., Kim, S. M., Zhao, Y., Hwang, H., Miura, S. K., Sossin, W. S., et al. (2009). Synapse- and stimulus-specific local translation during long-term neuronal plasticity. Science 324, 1536-1540. doi: 10.1126/science.1173205

Werner, A. (2013). Biological functions of natural antisense transcripts. BMC Biol. 11:31. doi: 10.1186/1741-7007-11-31

Wight, M., and Werner, A. (2013). The functions of natural antisense transcripts. Essays Biochem. 54, 91-101. doi: 10.1042/bse0540091

Yoshigai, E., Machida, T., Okuyama, T., Mori, M., Murase, H., Yamanishi, R., et al. (2013). Citrus nobiletin suppresses inducible nitric oxide synthase gene expression in interleukin-1beta-treated hepatocytes. Biochem. Biophys. Res. Commun. 439, 54-59. doi: 10.1016/j.bbrc.2013.08.029

Zhang, Y., Li, J., Kong, L., Gao, G., Liu, Q. R., and Wei, L. (2007). NATsDB: natural antisense transcripts database. Nucleic Acids Res. 35, D156-D161. doi: $10.1093 / \mathrm{nar} / \mathrm{gkl} 782$

Conflict of Interest Statement: The authors declare that the research was conducted in the absence of any commercial or financial relationships that could be construed as a potential conflict of interest.

Received: 30 November 2013; accepted: 29 March 2014; published online: 22 April 2014.

Citation: Kadakkuzha BM, Liu X-A, Narvaez M, Kaye A, Akhmedov $K$ and Puthanveettil SV (2014) Asymmetric localization of natural antisense RNA of neuropeptide sensorin in Aplysia sensory neurons during aging and activity. Front. Genet. 5:84. doi: 10.3389/fgene.2014.00084

This article was submitted to Non-Coding RNA, a section of the journal Frontiers in Genetics.

Copyright (c) 2014 Kadakkuzha, Liu, Narvaez, Kaye, Akhmedov and Puthanveettil. This is an open-access article distributed under the terms of the Creative Commons Attribution License (CC BY). The use, distribution or reproduction in other forums is permitted, provided the original author(s) or licensor are credited and that the original publication in this journal is cited, in accordance with accepted academic practice. No use, distribution or reproduction is permitted which does not comply with these terms. 